\title{
Statistical scaling of pore-scale Lagrangian velocities in natural porous media
}

\author{
M. Siena, ${ }^{1, *}$ A. Guadagnini, ${ }^{1,2}$ M. Riva,,${ }^{1,2}$ B. Bijeljic, ${ }^{3}$ J. P. Pereira Nunes,,${ }^{3,4}$ and M. J. Blunt ${ }^{1,3}$ \\ ${ }^{1}$ Dipartimento di Ingegneria Civile e Ambientale, Politecnico di Milano, Piazza L. Da Vinci 32, 20133 Milano, Italy \\ ${ }^{2}$ Department of Hydrology and Water Resources, University of Arizona, Tucson, Arizona 85721, USA \\ ${ }^{3}$ Department of Earth Science and Engineering, Imperial College, London, United Kingdom \\ ${ }^{4}$ Reservoir Geophysics Group, Petrobrás, Rio de Janeiro, Rio de Janeiro, Brazil
}

(Received 29 April 2014; revised manuscript received 2 July 2014; published 21 August 2014)

\begin{abstract}
We investigate the scaling behavior of sample statistics of pore-scale Lagrangian velocities in two different rock samples, Bentheimer sandstone and Estaillades limestone. The samples are imaged using x-ray computer tomography with micron-scale resolution. The scaling analysis relies on the study of the way $q$ th-order sample structure functions (statistical moments of order $q$ of absolute increments) of Lagrangian velocities depend on separation distances, or lags, traveled along the mean flow direction. In the sandstone block, sample structure functions of all orders exhibit a power-law scaling within a clearly identifiable intermediate range of lags. Sample structure functions associated with the limestone block display two diverse power-law regimes, which we infer to be related to two overlapping spatially correlated structures. In both rocks and for all orders $q$, we observe linear relationships between logarithmic structure functions of successive orders at all lags (a phenomenon that is typically known as extended power scaling, or extended self-similarity). The scaling behavior of Lagrangian velocities is compared with the one exhibited by porosity and specific surface area, which constitute two key pore-scale geometric observables. The statistical scaling of the local velocity field reflects the behavior of these geometric observables, with the occurrence of power-law-scaling regimes within the same range of lags for sample structure functions of Lagrangian velocity, porosity, and specific surface area.
\end{abstract}

DOI: 10.1103/PhysRevE.90.023013

PACS number(s): 47.56.+r, 47.61.-k, 89.75.Da, 91.60.Np

\section{INTRODUCTION}

Micron-resolution imaging of reservoir rock samples and associated flow and transport modeling is transforming our ability to characterize pore-scale processes in natural and reconstructed porous media (see, e.g., [1,2]). Simulations of flow through pore space images have determined that there is a billionfold variation in local flow velocities even across millimeter-size samples [3,4]. This can lead to highly anomalous (or non-Fickian) transport, which is supported by evidence from centimeter-scale experiments [3,5]. In this context, anomalous behavior denotes transport processes that cannot be described by an interpretation based on the classical advection-dispersion equation [6]. Such behavior arises from the complex and heterogeneous structure of natural porous media and is embedded in solute breakthrough curves characterized by early breakthrough and long tails as well as by non-Gaussian distributions of spatial displacements and of Lagrangian velocity increments (see [4] and references therein). Theoretical continuum-scale models for non-Fickian transport have been reviewed previously [6,7]. Recent research by Bijeljic et al. [3,4] presents experimental results, supported by numerical simulations, documenting the emergence of a relationship between pore structure, velocity distribution, and propagators (i.e., solute concentration vs displacement) in samples of real rocks. A connection of pore-structure heterogeneity with macroscopic flow and transport properties (e.g., permeability and dispersion coefficients) has been inferred from experimental results illustrated in recent works in the field of soil contamination and remediation problems [8,9].

A key aspect that has not been addressed in these studies is the assessment of the scaling behavior of static attributes of

*Corresponding author: martina.siena@polimi.it the pore space combined with related dynamic properties of the flow field taking place within the host rock. Indeed, the statistics of hydrological properties in aquifers and reservoirs exhibit dependences on various characteristic length scales, e.g., measurement volume and resolution, scale of observation, spatial correlation, and size of the sampled domain [10]. Scaling analyses of statistical moments of hydraulic conductivity and permeability data have been presented in many works, including [11-20]. In all these studies, measurement volumes are associated with a support scale ranging from the millimeter to the meter and data sets are collected on domains at the field (kilometer) or laboratory (decimeter to meter) scale. Recently, Guadagnini et al. [21] documented and analyzed the statistical scaling of geometric observables, i.e., porosity and specific surface area, of two different rocks at the millimeter scale.

In this work we investigate the scaling of increments, or changes, in local flow velocity across a range of lengths, spanning from the (micron) resolution scale of the rock images up to the (millimeter) scale of the sample sizes. Similar to [11-21], we do so by relying on the analysis of sample structure functions of order $q>0$ of such increments. We then compare the scaling behaviors observed for static (i.e., local porosity and surface area) and dynamic (i.e., Lagrangian velocity) quantities, evaluated on the same rock samples. Our work is an initial step towards the quantification of statistical scaling of dynamic variables involved in transport processes and provides key basic elements that are required to translate a detailed statistical analysis at the micron scale to (statistical) moments of flow (and possibly transport) on larger investigation scales typical of oil reservoirs and aquifers. The results of this analysis can be of primary interest in all fields where the heterogeneous and multiscale nature of geological media plays a key role, including, e.g., oil and gas engineering applications, groundwater hydrology, geophysics, and petrophysics. 
(a)

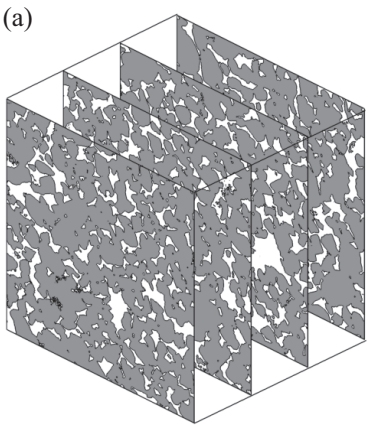

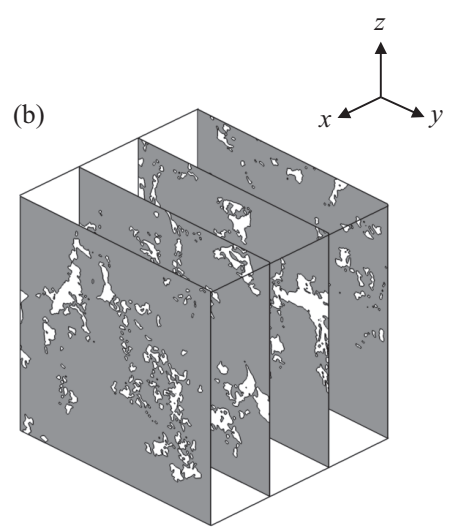

FIG. 1. Images of the void space (white) along cross-sectional planes orthogonal to the mean flow direction ( $x$ axis) for (a) Bentheimer sandstone and (b) Estaillades limestone.

\section{DATA SETS AND THEORETICAL FRAMEWORK}

\section{A. Pore-space imaging}

We perform our analysis on samples of two different rocks: (i) Bentheimer sandstone and (ii) Estaillades limestone. The three-dimensional structures of the pore spaces of the two samples are reconstructed via X-ray computer microtomography (XCT) using a bench-top scanner (Xradia Versa 500) with spatial resolution $l=3.0$ and $3.3 \mu \mathrm{m}$ for the sandstone and the limestone, respectively. Technical details concerning XCT reconstruction can be found in [21] and references therein. We produce images that are cubic arrays of ones and zeros, respectively representing solid and pore space. Bentheimer and Estaillades images are respectively composed of $1000^{3}$ and $650^{3}$ voxels. The overall image domain is also a cube of side length $3 \mathrm{~mm}$ for Bentheimer and $2.145 \mathrm{~mm}$ for Estaillades.

Figure 1 depicts images of the pore space within the two samples, across selected cross-sectional planes orthogonal to the mean flow direction, which we identify as the $x$ axis. Table I lists key features of the two cubic samples: voxel array size $N_{\text {vox }}$, image resolution $l$, overall side length $L$, total

TABLE I. Main geometrical features of the Bentheimer sandstone and Estaillades limestone samples: voxels array size $N_{\text {vox }}$, image resolution $l$, overall side length $L$, porosity $\phi$, and specific surface area $\mathcal{S}$. Here $N_{P}$ is the number of pathways extending at least to $90 \%$ of the whole domain along the $x$ axis.

\begin{tabular}{lcccccc}
\hline \hline Sample & $N_{\text {vox }}$ & $l(\mu \mathrm{m})$ & $L(\mu \mathrm{m})$ & $\phi$ & $\mathcal{S}\left(\mu \mathrm{m}^{-1}\right)$ & $N_{P}$ \\
\hline Bentheimer sandstone & $1000^{3}$ & 3.0 & 3000 & 0.22 & 0.07 & 7014 \\
Estaillades limestone & $650^{3}$ & 3.3 & 2145 & 0.12 & 0.04 & 2850 \\
\hline \hline
\end{tabular}

porosity $\phi$ (i.e., fraction of total volume occupied by pores), and specific surface area $\mathcal{S}$ (i.e., interfacial area between pores and solid matrix per unit volume). For the purpose of our scaling analyses presented in Sec. III, we follow Guadagnini et al. [21] and compute the distributions of porosity $\phi$ and $\mathcal{S}$ along $x$ (represented in Fig. 1), associated with rock volumes of size $L^{2} \times l$ by applying the methodology of Coker and Torquato [22] to the digitized binary images.

\section{B. Flow and transport simulations within the pore space}

Lagrangian velocities have been obtained by tracking the trajectories of a large number-O $\left(10^{4}\right)$ —of particles through the steady-state flow fields computed by way of pore-scale numerical simulations within both rock samples. The NavierStokes equations for single-phase incompressible flow are solved in the pore space by the method presented in [3,23], which is based on the finite-volume code in OpenFOAM [24]. A finite-volume mesh is generated by converting each image voxel into a grid block. Fluid flow is simulated from one face to the opposite face of the system. Boundary conditions are assigned by assuming constant pressure and zero-gradient velocities at the inlet and outlet faces and no-flow conditions on the lateral sides. A no-slip boundary condition is used at solid voxel boundaries. Pressure and velocity values are obtained by solving mass and momentum balance equations through an iterative scheme based on the PISO algorithm [25], until the velocity field attains a steady-state configuration. Figure 2 depicts the resulting steady-state velocity fields for Bentheimer (a)

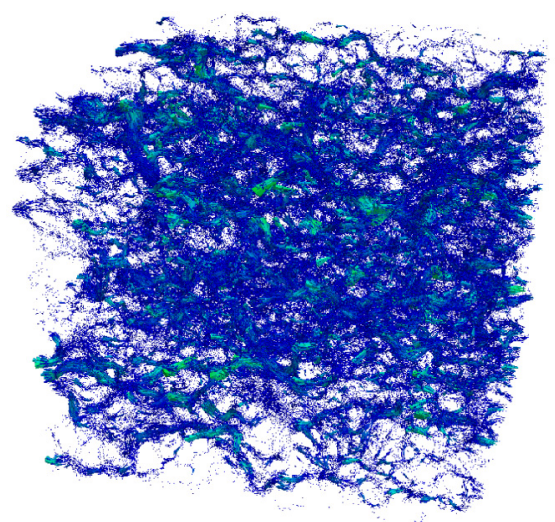

(b)

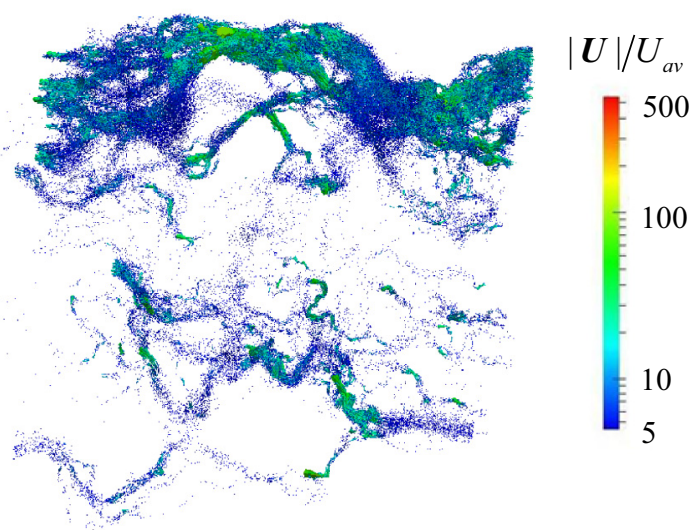

FIG. 2. (Color online) Computed normalized flow fields for (a) Bentheimer sandstone and (b) Estaillades limestone. Results are depicted as the ratios of the magnitude of velocity $|\boldsymbol{U}(\boldsymbol{x})|$ at the voxel centers divided by the average pore velocity magnitude $U_{a v}$ and represented on a logarithmic colorized scale (average flow is from left to right). 
and Estaillades (only velocities values that are at least five times higher than the average pore velocity are shown, similar to the analysis in [4]) to illustrate the differences in the spatial structure of the fast flow lines in the two systems.

The particle trajectories and times of flight are then computed semianalytically by tracing streamlines through the pore space $[26,3,4]$, corresponding to simulating advectiondominated displacement in the pore space. The starting point of each trajectory is chosen randomly within the pore space and the particle is tracked until it leaves the domain or until the total travel time exceeds $10^{6} \mathrm{~s}$ (note that the travel time at the average calculated flow velocity is less than $10 \mathrm{~s}$ in both sample rocks). The time of passage, spatial coordinates, and velocity components along the three Cartesian axes $\boldsymbol{x} \equiv$ $(x, y, z)$ are registered whenever a particle crosses the faces of a voxel. Among all particles injected, we select those pathways spanning at least $90 \%$ of the whole domain along the mean flow direction (identified by the $x$ axis in Fig. 1). The total number of particles $N_{P}$ satisfying this requirement for each rock sample is also listed in Table I.

\section{Scaling of statistics}

Following [11-21], statistical scaling of moments of velocity is investigated through the analysis of directional sample structure functions, i.e., $q$ th-order statistical moments of absolute increments

$$
S_{N}^{q}\left(s_{i}\right)=\frac{1}{N\left(s_{i}\right)} \sum_{n=1}^{N\left(s_{i}\right)}\left|\Delta Y_{n}\left(s_{i}\right)\right|^{q},
$$

where $\Delta Y_{n}\left(s_{i}\right)$ is a computed (or measured) increment along the $i$ axis (with $i=x, y, z), \Delta Y\left(s_{i}\right)=Y\left(\boldsymbol{x}+s_{i}\right)-Y(\boldsymbol{x})$, of the random field $Y(\boldsymbol{x})$, evaluated along the directional separation distance (or lag) $s_{i}$. It can be useful to point out that $S_{N}^{2}$, i.e., the structure function of order $q=2$, is related to the variogram of $Y(\boldsymbol{x})$, which forms the basis for standard analyses of random fields. The field $Y(\boldsymbol{x})$ is said to exhibit power-law scaling if

$$
S_{N}^{q}\left(s_{i}\right) \sim\left(s_{i}\right)^{\xi(q)}
$$

Here the scaling exponent $\xi(q)$ depends only on the order $q$. When $\xi(q)$ displays a linear dependence on $q$, i.e., $\xi(q)=H q$ [ $H$ being a Hurst exponent, which is an index of the degree of (spatial) persistence of the signal], the field $Y$ is considered to constitute a self-affine (monofractal) random field. When $\xi(q)$ depends in a nonlinear fashion on $q, Y$ has traditionally been considered to be multifractal (or to be interpreted as fractional Laplace motion). The reader is referred to [27] for a recent discussion on these topics.

Similar to [21], we investigate the power-law-scaling behavior of Eq. (1), for different orders $q$, by means of two methods: (a) method of moments (MM) and (b) extended self-similarity (ESS).

The method of moments aims at assessing the occurrence of a power-law dependence of sample structure functions on lag $s_{i}$ of the kind expressed by Eq. (2). Such scaling behavior is typically limited to an intermediate range of lags $s_{I} \leqslant s_{i} \leqslant s_{I I}$ and has been observed for a broad variety of environmental, geophysical, and other variables (including hydraulic, hydrogeological, biological, astrophysical, ecological, and physical). Identification of the exponent $\xi(q)$ enables one to characterize the way statistical moment transition with scale. Power-law behavior is inferred by means of linear regression applied on the log-log plots of $S_{N}^{q}\left(s_{i}\right)$ vs $s_{i}$, in the case of the MM.

On the other hand, extended power-law scaling (or ESS) arises from the empirical observation of the occurrence at all lags of linear dependence of logarithmic structure functions of successive orders. In this sense, ESS enables one to extend the scaling range to the complete range of separation scales examined, as shown empirically by [28-30] and references therein, and provides another way to characterize the dependence of the scaling exponent $\xi(q)$ on $q$. Extended self-similarity is described by the following power-law relationship between sample structure functions of different orders [31,32]

$$
S_{N}^{p}\left(s_{i}\right) \sim\left[S_{N}^{q}\left(s_{i}\right)\right]^{\beta(p, q)} .
$$

Here $\beta(p, q)=\xi(p) / \xi(q)$, i.e., the ratio between scaling exponents of $S_{N}^{p}$ and $S_{N}^{q}$ defined in Eq. (2). With ESS, power-law behavior is inferred by means of linear regression applied on the log-log plots of $S_{N}^{p}\left(s_{i}\right)$ vs $S_{N}^{q}\left(s_{i}\right)$. The scaling exponents $\xi(q)$ and $\beta(p, q)$ are evaluated as the slopes of the regression lines.

It has been shown in the literature that the breakdown in the power-law scaling defined in Eq. (2), which is typically observed at small $\left(s_{i} \leqslant s_{I}\right)$ and large lags $\left(s_{i} \geqslant s_{I I}\right)$, is consistent with the view of $Y(\boldsymbol{x})$ as a sample from a truncated fractional Brownian motion (TFBM), truncated fractional Gaussian noise (TFGN), or a sub-Gaussian random field subordinated to TFBM or TFGN (e.g., [27]). According to these theoretical models, $Y(\boldsymbol{x})$ represents a hierarchy of superimposed, statistically homogeneous, multivariate, Gaussian, or sub-Gaussian random fields. The hierarchy is truncated from below by a cutoff proportional to the measurement or resolution length scale of the data and from above by a cutoff proportional to the characteristic scale of the sampling domain. These cutoffs are intimately related to the breakdown in power-law scaling. Interpretations based on such modeling framework have been proven to be consistent with observations associated with a variety of laboratory and field scale hydrological and pedological data from sedimentary and fractured rocks [17-20,33]. With reference to ESS, a theoretical basis for Eq. (3) and its validity for all investigated lags has been provided with reference to (a) the one-dimensional Burger equation [34], (b) TFBM or TFGN [18], and (c) sub-Gaussian random processes subordinated to TFBM or TFGN [27].

\section{SCALING OF LAGRANGIAN VELOCITIES}

We compute increments of the $x$ component of Lagrangian velocity $U_{x}(\boldsymbol{x})$ along each trajectory for distances $s_{x}$ traveled by a particle along the $x$ direction (see Fig. 1 for the Cartesian frame of reference). We consider values of $s_{x}$ that are integer multiples of the image resolution $l$ and within the range $l \leqslant s_{x} \leqslant L / 2$. Sample structure functions $S_{N}^{q}\left(s_{x}\right)$ of absolute increments $\left|\Delta U_{x}\right|$ are computed according to Eq. (1) by considering jointly incremental data for all of the trajectories. Each point of $S_{N}^{q}\left(s_{x}\right)$ is then calculated on the basis of a very large 

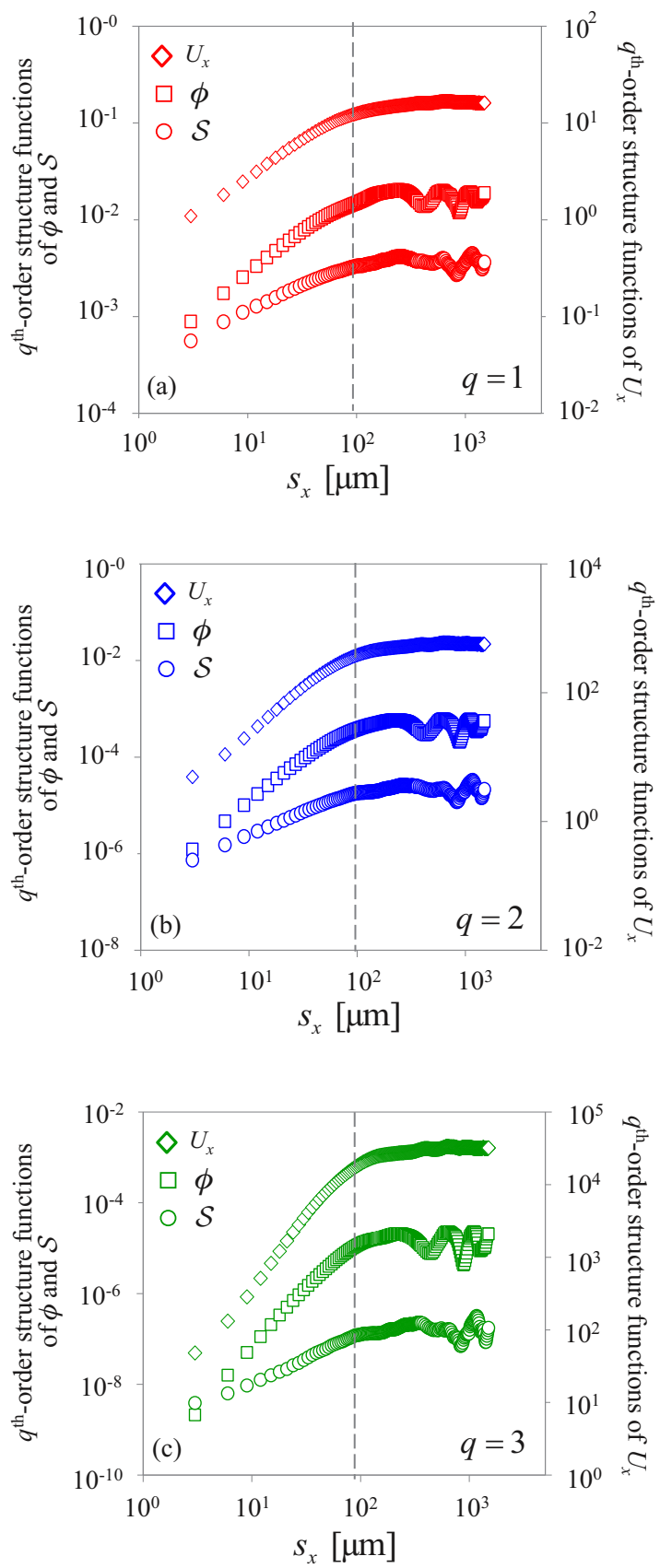

FIG. 3. (Color online) Sample structure functions of absolute increments of $\phi(x), \mathcal{S}(x)$, and $U_{x}(\boldsymbol{x})$ computed at lags $s_{x}$ along the $x$ axis for the Bentheimer sandstone and (a) $q=1$, (b) $q=2$, and (c) $q=3$.

number of statistical samples, i.e., $N\left(s_{x}\right)=O\left(10^{6}-10^{7}\right)$ for the Bentheimer sandstone and $N\left(s_{x}\right)=O\left(10^{5}-10^{6}\right)$ for the Estaillades limestone. The scaling analysis of the static $[\phi(x)$ and $\mathcal{S}(x)]$ and dynamic $\left[U_{x}(\boldsymbol{x})\right]$ properties of the two rocks is presented in Secs. III A and III B. Following [21], the quantities $\phi$ and $\mathcal{S}$ are calculated as averages over all cross sections orthogonal to the direction of the mean flow ( $x$ axis) and are therefore functions only of the spatial coordinate $x$. Sample structure functions of (absolute) spatial increments of $\phi$ and $\mathcal{S}$ are computed for the same $s_{x}$ values employed for $\left|\Delta U_{x}\right|$.

\section{A. Bentheimer sandstone}

Sample structure functions of (absolute) incremental values of $U_{x}(\boldsymbol{x}), \phi(x)$, and $\mathcal{S}(x)$, computed for $q=1,2,3$, are illustrated in Figs. 3(a)-3(c), respectively. A power-law behavior associated with a limited range of lags can be recognized for all quantities (geometrical observables and velocities). All curves appear to have a tendency to flatten beyond the lag $s_{x} \approx 100 \mu \mathrm{m}$, which can be identified as the upper limit $s_{I I}$ beyond which power-law scaling breaks down. The observation of a common value of $s_{I I}$ for all quantities measured on the same sample is consistent with the interpretation of $s_{I I}$ as being proportional to the size of the sampling domain, according to the theoretical framework of Neuman et al. [27]. On the other hand, from a qualitative inspection, it is hard to identify unambiguously a value for a lower limit $s_{I}$ of the scaling range for the curves analyzed. This observation is also consistent with the interpretation of [27], where $s_{I}$ is viewed as proportional to the measurement scale, which can vary with the type of data considered.

We start by analyzing scaling of sample structure functions of Lagrangian velocities through the MM. Figure 4(a) depicts, on a $\log -\log$ scale, the dependence of sample structure functions of velocity $S_{N}^{q}$ on $s_{x}$ for integer orders $1 \leqslant q \leqslant$ 4. According to the MM, power-law-scaling behavior can be identified in the intermediate range of lags $s_{I} \leqslant s_{x} \leqslant s_{I I}$ within which the log-log regression line fitted to the data is associated with the highest coefficient of determination $R^{2}$. We found (with $R^{2} \geqslant 0.98$ for all orders $q$ ) $s_{I}=3 \mu \mathrm{m}$ and $s_{I I}=99 \mu \mathrm{m}$, as indicated by dashed lines in Fig. 4(a).

As an example of results that can be obtained by ESS, Fig. 4(b) depicts, on a log-log scale, the way $S_{N}^{q+1}\left(s_{x}\right)$ varies with $S_{N}^{q}\left(s_{x}\right)$ for $1 \leqslant q \leqslant 4$. The straight-line relationships displayed by $\log -\log$ plots of $S_{N}^{q+1}$ versus $S_{N}^{q}$ demonstrate that the data analyzed satisfy ESS with high confidence $\left(R^{2} \approx 1.0\right.$ for all orders $q$ ). Equations of the power-law fits are also shown in Fig. 4(b) for completeness. Similar to what is observed for the scaling behavior evidenced above through the $\mathrm{MM}$, we consider also these types of results to be consistent with the interpretation proposed by [27], which can explain the ESS of variables that do not necessarily satisfy Burger's equation [34].

We then investigate functional relationships between the power exponent $\xi(q)$ and the order $q$ of structure functions within the identified scaling regime. As indicated in Sec. II C, according to the MM, these powers are the slopes of regression lines fitted to $\log -\log$ plots of $S_{N}^{q}\left(s_{x}\right)$ versus $s_{x}$, such as those depicted in Fig. 4(a). In the case of ESS, we (i) evaluate $\beta(q+\Delta q, q)=\xi(q+\Delta q) / \xi(q)$, for given $\Delta q$, as the slope of the regression line fitted to log-log plots of $S_{N}^{q+\Delta q}\left(s_{x}\right)$ versus $S_{N}^{q}\left(s_{x}\right)$ such as those depicted in Fig. 4(b); (ii) compute by the $\mathrm{MM}$ a reference value $\xi_{\text {ref }}$ of $\xi(q)$ for a selected $q=q_{\text {ref }}$; and (iii) start from $\xi_{\text {ref }}$ to evaluate $\xi(q+\Delta q)$ and $\xi(q-\Delta q)$ according to $\xi(q+\Delta q)=\beta(q+\Delta q, q) \xi(q)$ and $\xi(q-\Delta q)=\xi(q) / \beta(q, q-\Delta q)$. Following [21] and references therein, we adopt $\xi_{\text {ref }}=\xi(1)$.

Plots of $\xi(q)$ as a function of $q$, evaluated by the method of moments and ESS are presented in Fig. 5. Results obtained by the two methods are very similar. In all cases $\xi(q)$ delineates convex functions that lie below the straight line having slope 

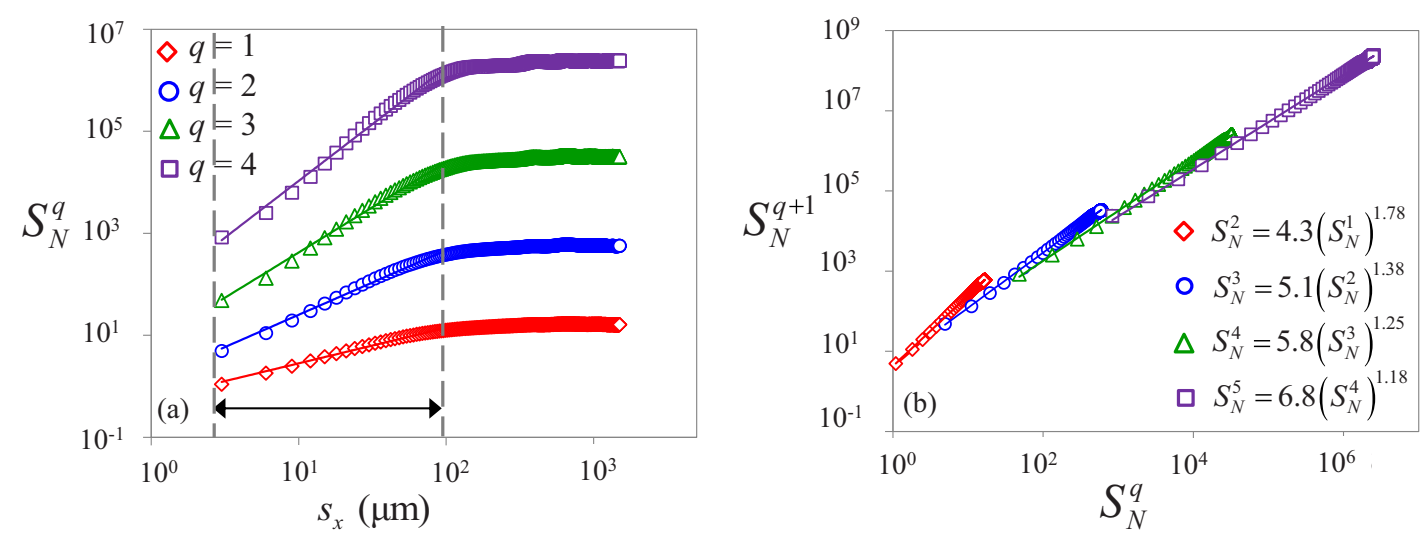

FIG. 4. (Color online) (a) Sample structure functions $S_{N}^{q}\left(s_{x}\right)$ of absolute increments of $U_{x}$ versus $s_{x}$ and (b) $S_{N}^{q+1}$ versus $S_{N}^{q}$ for the Bentheimer sandstone and $q=1,2,3,4$. Vertical dashed lines demarcate breaks in power-law-scaling regimes. Logarithmic scale regression lines indicating extended power-law scaling and the corresponding relations between $S_{N}^{q+1}$ versus $S_{N}^{q}$ are given in (b).

approximately equal to 0.72 and passing through the origin. This slope is typically regarded in the literature as an estimate of the Hurst exponent $H$. Similar to the scaling behavior documented through the MM and ESS, also the types of behavior exhibited by $\xi(q)$ in Fig. 5 can be considered to be consistent with the interpretation proposed by [27].

\section{B. Estaillades limestone}

Guadagnini et al. [21] investigated the statistical scaling of $\phi$ and $\mathcal{S}$ on the same sample of Estaillades limestone we analyze. Here we analyze jointly their results [see Figs. 2(b) and 3(b) of [21]] and those stemming from sample structure functions of Lagrangian velocity increments. Figures 6(a)-6(c) present $\log$-log depictions of $S_{N}^{q}\left(s_{x}\right)$ versus $s_{x}$ of (absolute) increments of $U_{x}(\boldsymbol{x}), \phi(x)$, and $\mathcal{S}(x)$ for $q=1,2$, and 3 , respectively. It can be noted that all plots show a linear increase (corresponding to a power-law dependence of $S_{N}^{q}$ on $s_{x}$ ) up to a lag $\bar{s}_{x} \approx 40 \mu \mathrm{m}$, this upper limit being identified by a dashed line in the figures. For $s_{x}>\bar{s}_{x}$ a linear increase associated with a different slope can be recognized. This behavior can be explained by two diverse arguments: (a) The change of the

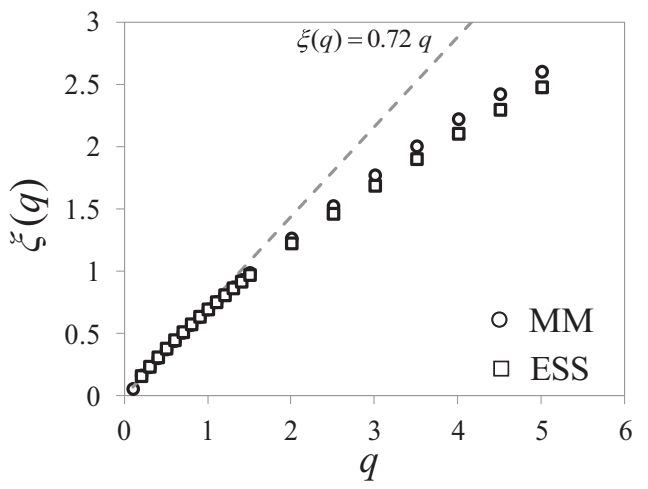

FIG. 5. Scaling exponent $\xi(q)$ evaluated as a function of $q$ by the MM and ESS for the Bentheimer sandstone sample. The slope of the dashed straight line passing through $\xi(1)$ and the origin (equation listed) provides an estimate of the Hurst exponent. slope of the linear trends is due to the effect of a lower limit of the scaling region $s_{I}=\bar{s}_{x} \approx 40 \mu \mathrm{m}$ above which power-law scaling takes place or (b) the two slopes reveal the occurrence of two power-law-scaling regimes. The latter behavior could be interpreted as the effect of two underlying structures that are characterized by different spatial correlations.

In order to analyze the relative robustness of these interpretations, we also explore the statistical scaling of the geometrical features of the sample on the basis on an XCT reconstruction characterized by a coarser resolution $l^{\prime}=$ $7 \mu \mathrm{m}$. Figure 7 depicts sample structure functions of absolute increments of $\phi(x)$ [Fig. 7(a)] and $\mathcal{S}(x)$ [Fig. 7(b)] associated with low $\left(l^{\prime}=7 \mu \mathrm{m}\right)$ and high $(l=3.3 \mu \mathrm{m})$ resolution. Sample structure functions obtained within the low-resolution domain exhibit a single power-law-scaling regime, within the range $s_{I}\left(=l^{\prime}=7 \mu \mathrm{m}\right) \leqslant s_{x} \leqslant s_{I I}(=300 \mu \mathrm{m})$, whereas the curves tend to flatten for $s_{x}>s_{I I}$. These results are not consistent with our hypothesis (a), according to which the effect of the lower cutoff should be amplified by increasing the degree of spatial resolution, the theoretical value of $s_{I}$ being proportional to the resolution scale $l^{\prime}$. These results are consistent with our interpretation (b), suggesting that the power-law behavior observed in the range of small lags (i.e., $s_{x}<\bar{s}_{x} \approx 40 \mu \mathrm{m}$ ) within the high-resolution domain is due to a spatially correlated structure that cannot be resolved by the low-resolution XCT reconstruction. To further support this picture, we calculate estimates of the Hurst exponents for porosity $\hat{H}_{\phi}$ and specific surface area $\hat{H}_{\mathcal{S}}$, following the procedure illustrated in Sec. III A, relying on the method of moments. For the low-resolution domain we estimate $\hat{H}_{\phi}=0.68$ and $\hat{H}_{\mathcal{S}}=0.77$ in the range $s_{I}\left(=l^{\prime}=7 \mu \mathrm{m}\right) \leqslant$ $s_{x} \leqslant s_{I I}(=300 \mu \mathrm{m})$. As mentioned above, we identify two distinct power-law-scaling regimes, respectively indicated as scaling regions I and II, in the high-resolution domain. These regimes are characterized by different estimates of the Hurst scaling exponent for $\phi$ and $\mathcal{S}$, i.e., $\hat{H}_{\phi}^{(I)}=0.86$ and $\hat{H}_{\mathcal{S}}^{(I)}=$ 0.56 in the scaling range associated with small lags (region I) $s_{I}(=3.3 \mu \mathrm{m}) \leqslant s_{x} \leqslant \bar{s}_{x}(=40 \mu \mathrm{m})$ and $\hat{H}_{\phi}^{(I I)}=0.68$ and $\hat{H}_{\mathcal{S}}^{(I I)}=0.75$ in the scaling range $\bar{s}_{x} \leqslant s_{x} \leqslant s_{I I}(=300 \mu \mathrm{m})$ 

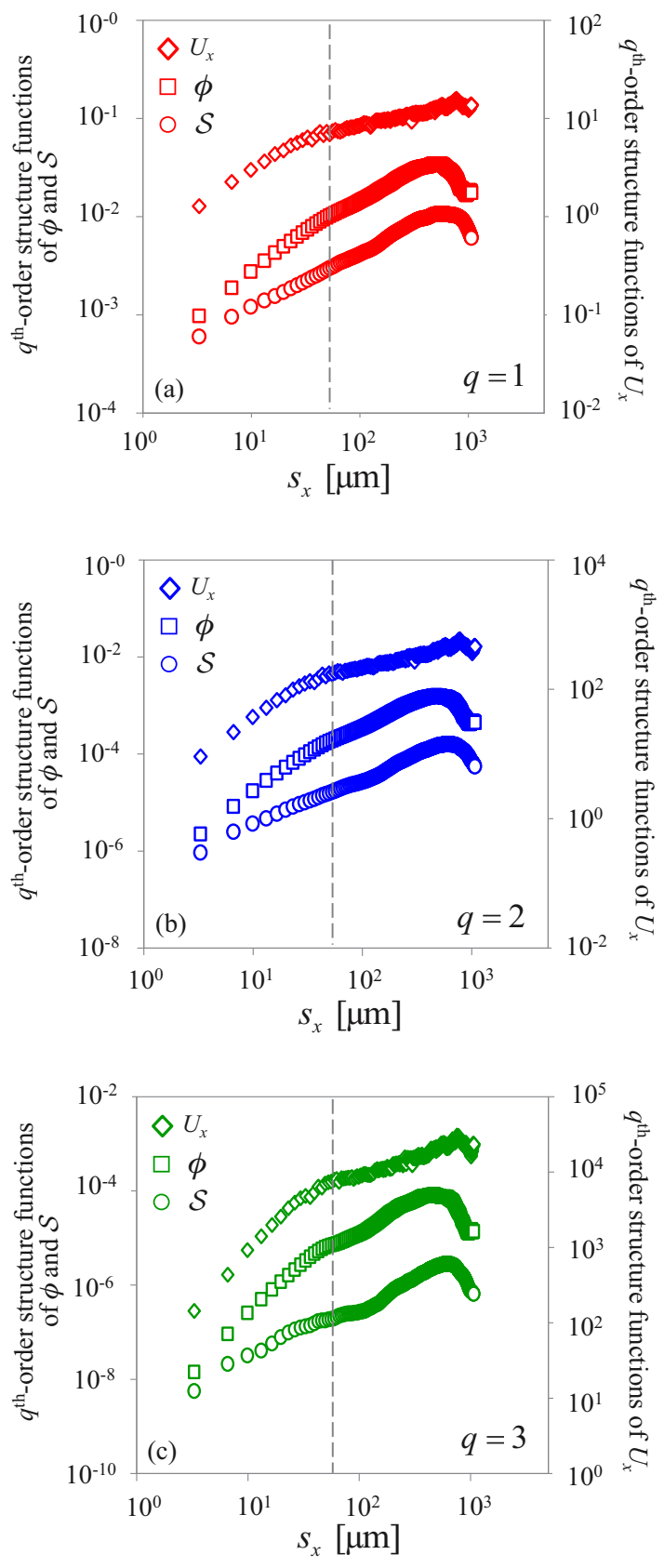

FIG. 6. (Color online) Sample structure functions of absolute increments of $\phi(x), \mathcal{S}(x)$, and $U_{x}(\boldsymbol{x})$ computed at lags $s_{x}$ along the $x$ axis for the Estaillades limestone and (a) $q=1$, (b) $q=2$, and (c) $q$ $=3$.

(region II). We note that these values are all indicative of a mild to strong spatial persistence (i.e., tendency of large and small values of the variable to alternate mildly in space within the system) of the signal analyzed and $\hat{H}_{\phi}^{(I I)}$ and $\hat{H}_{\mathcal{S}}^{(I I)}$ are very similar to their counterparts obtained in the lowresolution domain. These results are consistent with the observation that an increased level of resolution reveals the emergence of an additional correlation structure that is likely to be associated with microporosity in the pore structure and affects the behavior of sample structure functions at small lags. This structure is not resolved with a coarser reconstruction
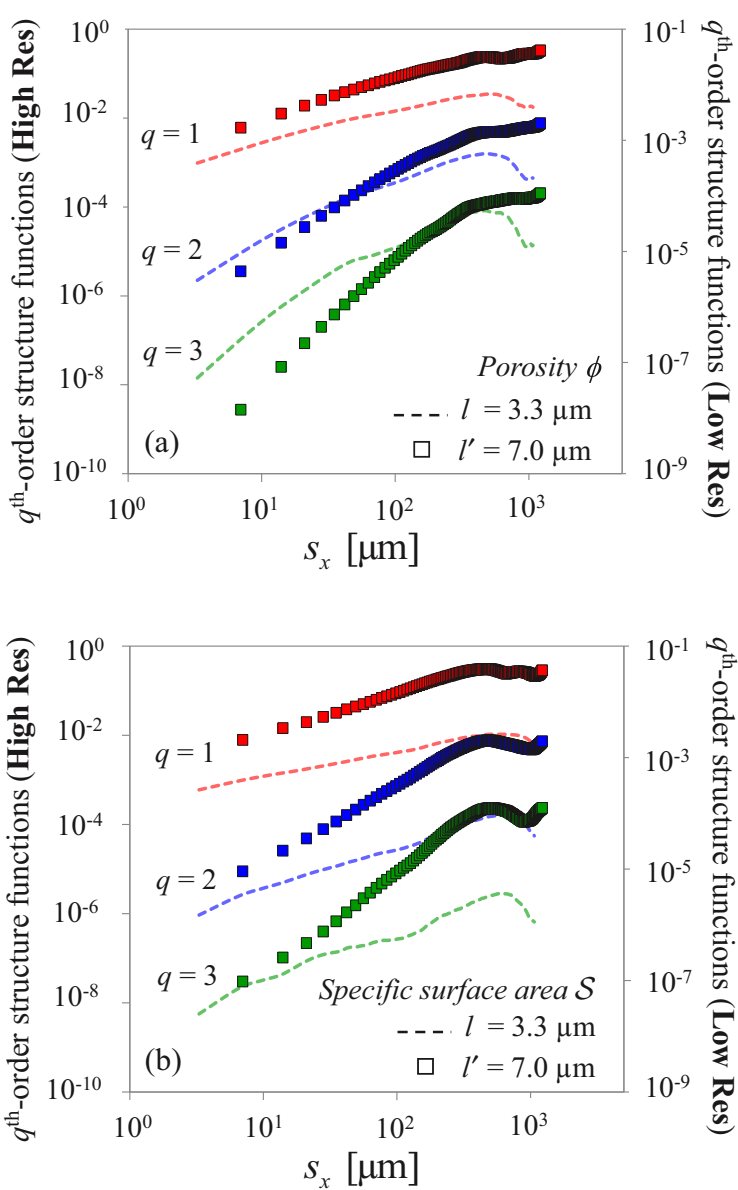

FIG. 7. (Color online) Sample structure functions of $\operatorname{order} q=1$, 2, 3 of absolute increments of (a) $\phi(x)$ and (b) $\mathcal{S}(x)$ for two resolution levels of the XCT reconstruction, i.e., $l^{\prime}=7.0 \mu \mathrm{m}$ (labeled Low Res and plotted with symbols) and $l=3.3 \mu \mathrm{m}$ (labeled High Res and plotted as dashed lines).

of the rock sample. The scaling behavior associated with a correlation structure typical of large lags is captured in both low- and high-resolution domains.

On the basis of these considerations, we consider interpretation (b) to be more realistic and perform all scaling analyses associated with Lagrangian velocities within the high-resolution domain. Figures 8(a) and 8(b) illustrate the results obtained through the MM and ESS, respectively, depicting $\log -\log$ plots of $S_{N}^{q}$ of $\left|\Delta U_{x}\right|$ versus $s_{x}$ [Fig. 8(a)] and $S_{N}^{q+1}$ versus $S_{N}^{q}$ [Fig. 8(b)] for integer orders $1 \leqslant q \leqslant$ 4. Similarly to the results depicted in Figs. 7(a) and 7(b), Fig. 8(a) demonstrates the occurrence of the two distinct scaling regimes, labeled regions I and II, for sample structure functions of all orders. Figure 8(b) shows that, despite the two distinct scaling behaviors revealed by the MM, a unique power-law trend reflecting ESS behavior [Eq. (3)] is attained at all lags, with considerably high determination coefficients $\left(R^{2} \geqslant 0.99\right)$.

We then investigate the way the scaling exponent $\xi(q)$ varies with $q$ by means of both the MM and ESS. With reference to 

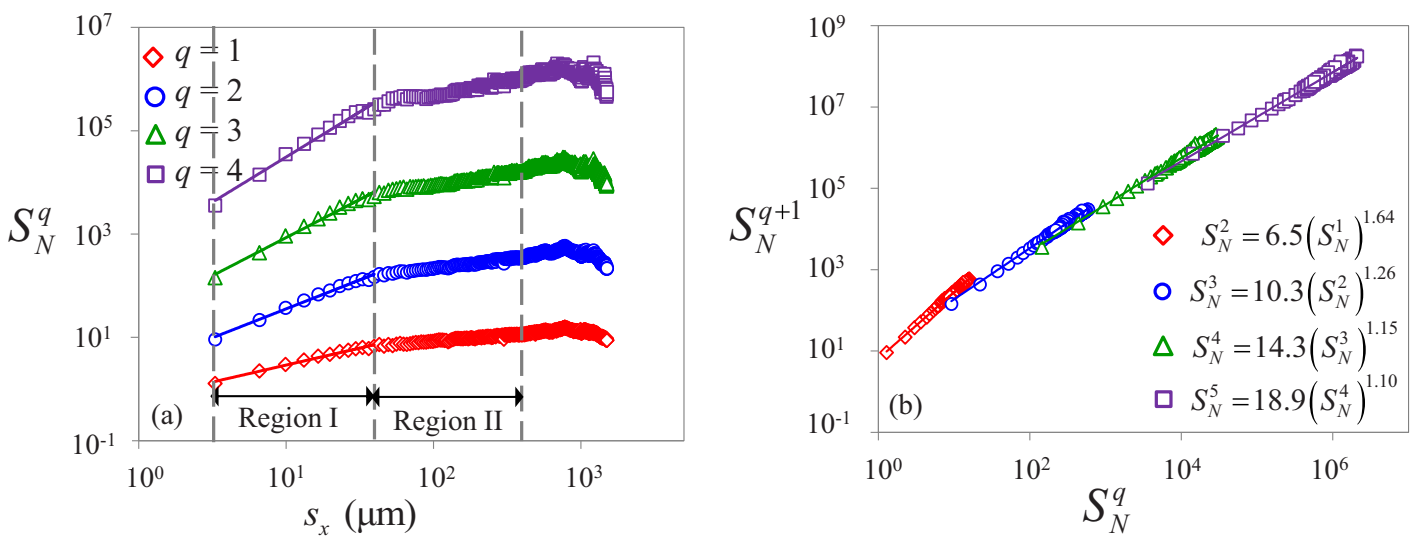

FIG. 8. (Color online) (a) Sample structure functions $S_{N}^{q}\left(s_{x}\right)$ of absolute increments of $U_{x}$ versus $s_{x}$ and (b) $S_{N}^{q+1}$ versus $S_{N}^{q}$ for the high-resolution Estaillades limestone and $q=1,2,3,4$. Vertical dashed lines demarcate the two identified power-law-scaling regimes. Logarithmic scale regression lines indicating extended power-law scaling and corresponding relations between $S_{N}^{q+1}$ versus $S_{N}^{q}$ are given in (b).

ESS and considering Fig. 8(b), we note that

$$
\beta(q+\Delta q, q)=\frac{\xi_{I}(q+\Delta q)}{\xi_{I}(q)}=\frac{\xi_{I I}(q+\Delta q)}{\xi_{I I}(q)},
$$

where $\xi_{I}(q)$ and $\xi_{I I}(q)$ are the slopes of the regression lines fitted to the sample structure function of a given order $q$ within regions I and II, respectively. The functions $\xi_{I}(q)$ and $\xi_{I I}(q)$ based on ESS are calculated by assuming $\xi_{I, \text { ref }}=\xi_{I}(1)$ and $\xi_{I I, \text { ref }}=\xi_{I I}(1)$. The results of the analysis are collected in Figs. 9(a) and 9(b) for regions I and II, respectively. Results obtained by the two methods are, for the most part, very similar. All curves associated with both scaling regimes display a linear dependence of $\xi(q)$ versus $q$ in the range $0.1 \leqslant q \leqslant 1$ and deviate from linearity for larger- $q$ values. Estimates of the Hurst exponent are $\hat{H}_{I}=0.67$ and $\hat{H}_{I I}=0.25$ for regions I and II, respectively, indicating that the correlated structure characterizing sample structure functions in the range of small lags has a higher degree of spatial persistency when compared against the underlying structure associated with large lags. This result is also consistent with the observation that $\hat{H}_{\phi}^{(I)}>\hat{H}_{\phi}^{(I I)}$. Similar to what is observed for the sandstone sample in
Sec. III A, we consider the observed scaling behavior to be consistent with the interpretation proposed by [27].

\section{CONCLUSIONS}

Our work leads to the following key conclusions.

(1) Sample structure functions (statistical moment of absolute increments) of the Lagrangian velocity component along the mean flow direction $U_{x}$ evaluated in two different samples of rocks reconstructed via x-ray computer tomography exhibit a clear power-law dependence on the spatial lag $s_{x}$ for intermediate ranges of lags. All scaling manifestations documented appear to be consistent with the interpretation proposed by [27], which is supported by theoretical $[35,36]$, computational [19,27,29,37], and extensive experimental $[17,18,20,21,33]$ evidence.

(2) For the Bentheimer sandstone sample, a single powerlaw regime is observed within a range of lags that spans more than one decade within the observation window (i.e., the size of the porous sample).

(3) For the Estaillades limestone sample, we recognize two distinct power-law trends attained on adjacent ranges of
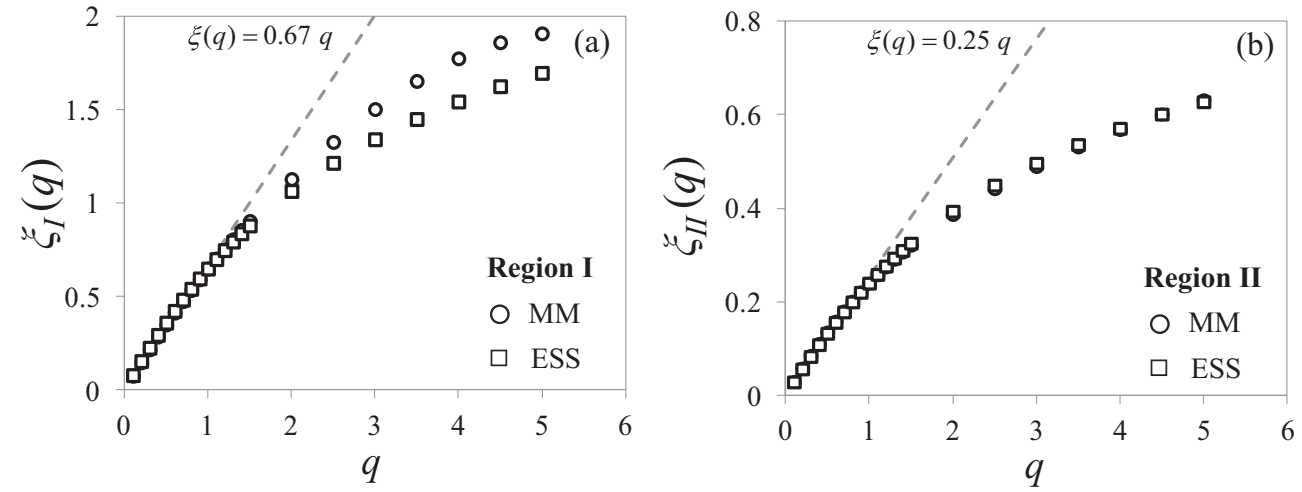

FIG. 9. Scaling exponent $\xi(q)$ evaluated as a function of $q$ by the MM and ESS for the high-resolution Estaillades limestone sample for scaling regions (a) I and (b) II. The slope of the dashed straight lines passing through $\xi(1)$ and the origin (equations listed) provides estimates of the Hurst exponents associated with the two scaling regimes. 
lags extending almost one decade each. We regard each of these power-law behaviors to represent an underlying spatially correlated structure. These distinct scaling regions emerge through analysis of high-resolution imaging and are likely to be associated with the effect of microporosity that is not captured at coarser levels of image resolution.

(4) For both rock samples, power-law scaling is extended at all lags by means of extended self-similarity. The method of moments and ESS provide scaling exponents $\xi(q)$ that vary linearly with $q$ for $0.1 \leqslant q \leqslant 1$ and deviate from linearity for $q>1$.

(5) Estimates of the Hurst exponents of $U_{x}$ indicate a higher degree of spatial persistence (tendency for large and small values not to alternate rapidly in space) in the Bentheimer sandstone than in the Estaillades limestone, consistent with the observation that the limestone is more heterogeneous in structure, flow, and transport [1,3,4]. Moreover, the spatial structure revealed in the limestone and associated with the range of small lags (identified as scaling region I) exhibits a higher degree of persistence when compared against the spatial structure associated with the largest lags (scaling region II).

(6) Statistical scaling of the local velocity (i.e., dynamic) field reflects the behavior of geometric (i.e., static) observables: Breakdown in power-law scaling, as well as the occurrence of different power-law-scaling regimes observed for the limestone sample, is exhibited within the same range of lags for sample structure functions of absolute increments of Lagrangian velocity, porosity, and specific surface area.

\section{ACKNOWLEDGMENTS}

Funding from MIUR (Italian Ministry of Education, Universities and Research Project "Innovative methods for water resources under hydro-climatic uncertainty scenarios") is acknowledged. J.P.P.N. gratefully acknowledges financial support from Petrobrás.
[1] M. J. Blunt, B. Bijeljic, H. Dong, O. Gharbi, S. Iglauer, P. Mostaghimi, A. Paluszny, and C. H. Pentland, Adv. Water Resour. 51, 197 (2013).

[2] D. Wildenschild and A. P. Sheppard, Adv. Water Resour. 51, 217 (2013).

[3] B. Bijeljic, A. Raeini, P. Mostaghimi, and M. J. Blunt, Phys. Rev. E 87, 013011 (2013).

[4] B. Bijeljic, P. Mostaghimi, and M. J. Blunt, Water Resour. Res. 49, 2714 (2013).

[5] B. Bijeljic, P. Mostaghimi, and M. J. Blunt, Phys. Rev. Lett. 107, 204502 (2011).

[6] B. Berkowitz, A. Cortis, M. Dentz, and H. Scher, Rev. Geophys. 44, RG2003 (2006).

[7] S. P. Neuman and D. M. Tartakovsky, Adv. Water Resour. 32, 670 (2009).

[8] C. D. Tsakiroglou and M. A. Ioannidis, Eur. J. Soil Sci. 59, 744 (2008).

[9] C. A. Aggelopoulos and C. D. Tsakiroglou, Geoderma 146, 412 (2008).

[10] S. P. Neuman and V. Di Federico, Rev. Geophys. 41, 1014 (2003).

[11] F. J. Molz and G. K. Boman, Geophys. Res. Lett. 22, 2545 (1995).

[12] H. H. Liu and F. J. Molz, Water Resour. Res. 33, 2483 (1997).

[13] F. J. Molz, H. H. Liu, and J. Szulga, Water Resour. Res. 33, 2273 (1997).

[14] A. Deshpande, P. B. Flemings, and J. Huang, J. Geophys. Res. 102, 15385 (1997).

[15] L. Tennekoon, M. C. Boufadel, D. Lavallee, and J. Weaver, Water Resour. Res. 39, 1193 (2003).

[16] J. W. Castle, F. J. Molz, S. Lu, and C. L. Dinwiddie, J. Sediment. Res. 74, 270 (2004).

[17] A. Guadagnini, S. P. Neuman, and M. Riva, Hydrol. Process. 26, 2894 (2012).
[18] M. Siena, A. Guadagnini, M. Riva, and S. P. Neuman, Hydrol. Earth Syst. Sci. 16, 29 (2012).

[19] M. Riva, S. P. Neuman, and A. Guadagnini, Stoch. Environ. Res. Risk Assess. 27, 195 (2013).

[20] M. Riva, S. P. Neuman, A. Guadagnini, and M. Siena, Vadose Zone J. 12, 1 (2013).

[21] A. Guadagnini, M. J. Blunt, M. Riva, and B. Bijeljic, Transp. Porous Med. 101, 465 (2014).

[22] D. A. Coker and S. Torquato, J. Appl. Phys. 77, 6087 (1995).

[23] A. Q. Raeini, M. J. Blunt, and B. Bijeljic, J. Comput. Phys. 231, 5653 (2012).

[24] OpenFOAM, The open source CFD toolbox, http://www.openfoam.com (2011).

[25] R. I. Issa, J. Comput. Phys. 62, 40 (1986).

[26] P. Mostaghimi, B. Bijeljic, and M. J. Blunt, SPE J. 17, 1131 (2012).

[27] S. P. Neuman, A. Guadagnini, M. Riva, and M. Siena, in Advances in Hydrogeology, edited by P. K. Mishra and K. L. Kuhlman (Springer, New York, 2013).

[28] V. I. Nikora and D. G. Goring, Math. Geol. 33, 251 (2001).

[29] A. Guadagnini and S. P. Neuman, Geophys. Res. Lett. 38, L13403 (2011).

[30] E. Leonardis, S. C. Chapman, and C. Foullon, Astrophys. J. 745, 185 (2012).

[31] R. Benzi, S. Ciliberto, C. Baudet, G. R. Chavarria, and R. Tripiccione, Europhys. Lett. 24, 275 (1993).

[32] R. Benzi, S. Ciliberto, R. Tripiccione, C. Baudet, F. Massaioli, and S. Succi, Phys. Rev. E 48, R29 (1993).

[33] A. Guadagnini, S. P. Neuman, M. G. Schaap, and M. Riva, Geoderma 214-215, 217 (2014).

[34] S. Chakraborty, U. Frisch, and S. S. Ray, J. Fluid Mech. 649, 275 (2010).

[35] S. P. Neuman, Hydrol. Process. 24, 2056 (2010).

[36] S. P. Neuman, Hydrol. Process. 25, 1837 (2011).

[37] S. P. Neuman, Geophys. Res. Lett. 37, L09403 (2010). 